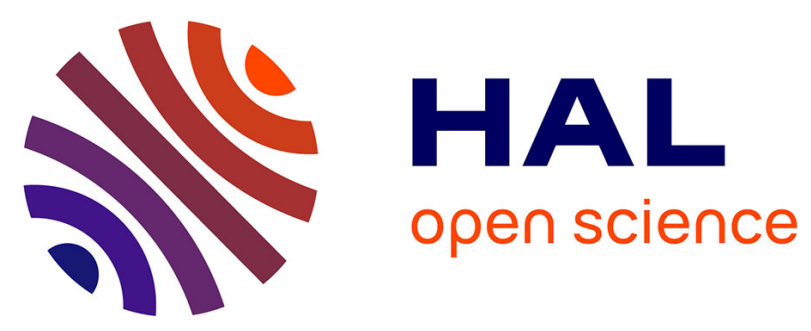

\title{
MOZART: Masking Outputs with Zeros for Architectural Robustness and Testing of DNN Accelerators
}

\author{
Stéphane Burel, Adrian Evans, Lorena Anghel
}

\section{- To cite this version:}

Stéphane Burel, Adrian Evans, Lorena Anghel. MOZART: Masking Outputs with Zeros for Architectural Robustness and Testing of DNN Accelerators. IEEE International On-Line Testing Symposium, Jun 2021, OnLine, France. hal-03470265

\section{HAL Id: hal-03470265 https://hal.science/hal-03470265}

Submitted on 8 Dec 2021

HAL is a multi-disciplinary open access archive for the deposit and dissemination of scientific research documents, whether they are published or not. The documents may come from teaching and research institutions in France or abroad, or from public or private research centers.
L'archive ouverte pluridisciplinaire $\mathbf{H A L}$, est destinée au dépôt et à la diffusion de documents scientifiques de niveau recherche, publiés ou non, émanant des établissements d'enseignement et de recherche français ou étrangers, des laboratoires publics ou privés. 


\title{
MOZART: Masking Outputs with Zeros for Architectural Robustness and Testing of DNN Accelerators
}

\author{
Stéphane Burel ${ }^{\dagger}$, Adrian Evans ${ }^{\dagger}$, Lorena Anghel ${ }^{\ddagger}$ \\ †Université Grenoble Alpes, CEA, LIST, Grenoble, France \\ ${ }^{\ddagger}$ Université Grenoble Alpes, CEA, CNRS, Grenoble INP*, INAC-Spintec \\ Email: \{stephane.burel,adrian.evans\}@cea.fr, lorena.anghel@grenoble-inp.fr
}

\begin{abstract}
Deep Neural Networks (DNNs) are increasingly used in safety critical autonomous systems. In this paper, we present MOZART, a DNN accelerator architecture which provides fault detection and fault tolerance. MOZART is a systolic architecture based on the Output Stationary (OS) variant, as it is the one that inherently limits fault propagation. In addition, MOZART achieves fault detection with on-line functional testing of the Processing Elements (PEs). Faulty PEs are swiftly taken off-line with minimal classification impact. The implementation of our approach on Squeezenet results in a loss of accuracy of less than $3 \%$ in the presence of a single faulty PE, compared to $15-33 \%$ without mitigation. The area overhead for the test logic does not exceed $8 \%$. Dropout during training further improves fault tolerance, without a priori knowledge of the faults.

Index Terms-neural network, accelerator, robustness, fault tolerance, convolution
\end{abstract}

\section{INTRODUCTION}

$\mathbf{T}$ HERE are many types of DNN accelerators, spanning GPUs, FPGAs and dedicated ASICs. Researchers are primarily focused on the design of accelerators optimized for performance and energy efficiency [1]. One of the most promising solutions used for the hardware implementation of DNNs are systolic architectures consisting of an array of processing elements (PEs) since they maximize local re-use of activations and weights [2], thus minimizing the costly data transfers with the external memory. These accelerators, if used in safety critical applications, have to show high robustness. Architectural fault-tolerance techniques can achieve robustness with minimal overhead.

Integrated circuits used in automotive applications must comply with ISO-26262. The most critical components need to detect and react to faults in an interval that is shorter than the fault tolerant time interval, the maximum time the fault can be present without posing a safety risk. As on-line testing becomes mandatory, new techniques must be developed that have lower overhead than traditional on-line testing.

Standards also impose stringent requirements on overall FIT (Failures in Time) rate (e.g. $\leq 10$ FIT for ASIL-D) which are difficult to achieve for large DNN circuits, as highlighted by [3]. Brute force approaches, such as Triple-Modular Redundancy (TMR) are costly and do not exploit the specificity of DNNs.

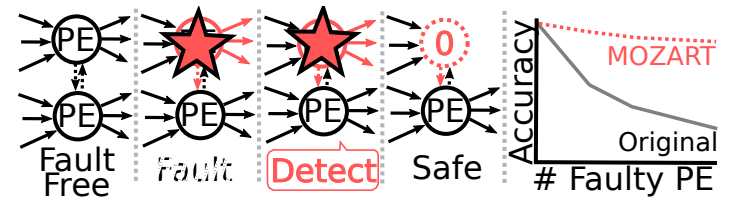

Fig. 1. MOZART Provides On-Line Testing and Fault Masking with Graceful Degradation in Accuracy

In this paper, we propose a new architecture called MOZART, which combines several new concepts which can be applied to provide fast on-line testing and fault mitigation for the data-path of a DNN accelerator.

MOZART relies on two major points : 1) During system operation, fault detection is performed at-speed in order to detect critical faults on the data-path; 2) An existing fault mitigation technique has been adapted to provide graceful degradation in classification accuracy, in the presence of faults, as illustrated in Figure 1. This can be augmented by a training technique which further increases robustness without a priori knowledge of the hardware faults.

The remaining sections are organized as follows. Sec. II presents our architecture. In Sec. III we define the case studies used in our experiments and then in Sec. IV we present the results. Sec. V presents related work on DNN fault tolerance and finally, we conclude with Sec. VI.

\section{MOZART ARCHITECTURE}

MOZART is a systolic Output Stationary (OS) DNN accelerator with data-path fault detection and mitigation. The dataflow in a DNN accelerator influences how faults propagate through the architecture towards the outputs and thus has a significant impact on the accuracy. We show that the OS architecture effectively contains the impact of fault, as a small number of neurons are impacted. A PE in an OS architecture roughly corresponds to a single neuron, thus making it possible to use an adaptation of the dropout technique to further improve the fault tolerance during the training phase. MOZART also exploits the fact that DNNs can obtain high accuracy even when certain intermediate calculations are masked to zero.

\section{A. Systolic Architectures}

Systolic architectures reduce memory transfers by reusing data. They consist of a fixed size array of PEs which perform 


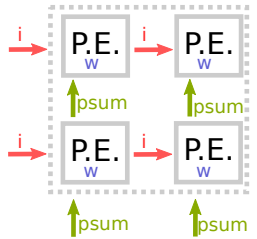

(a) WS Architecture

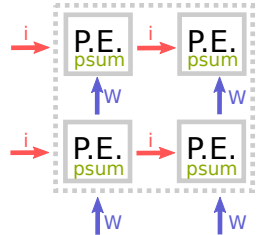

(b) OS Architecture

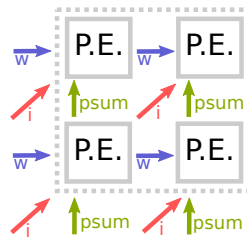

(c) RS Architecture
Fig. 2. Data-flow in Systolic DNN Accelerator Architectures

multiply-accumulate (MAC) operations and the PEs transfer data to their direct neighbours. The calculations of the abstract network are mapped to the PE array.

There are three broad classes of systolic architectures: weight, output and row stationary as shown in Figure 2. They rely on re-use of either weights, activations or both. We briefly present these architectures, however, the reader is referred to [2] for a full explanation.

1) Weight Stationary Data-flow: With a Weight Stationary (WS) data-flow [4], each column contains weights for a given output channel and performs the calculations for this channel. Weights are pre-loaded into the PEs and remain stationary. Activations flow horizontally and partial sums flow upwards. After several cycles, the sums arrive at the top of the column.

2) Output Stationary Data-flow: Alternatively, in an output stationary (OS) architecture (such as Shidiannao [5]), the partial sums stay fixed in the PE. The columns share weights, and rows share input features. Each PE is dedicated to a single output pixel. Weights and input features flow between the PEs.

3) Row Stationary Data-flow: In a row stationary (RS) architecture (such as Eyeriss [6]), 2-D convolutions are broken into 1-D convolutions, which are processed in a single PE. PEs store multiple weights for the same row and perform simultaneous MAC operations but only need storage of one partial sum. This architecture achieves re-use of both weights and partial sums. We consider a PE which stores 4 weights.

\section{B. Fault Detection and Mitigation}

With the MOZART approach, we introduce an on-line functional testing technique which ensures high coverage for impacting faults. During the testing procedure, each PE is taken off-line, one at a time. The PE's outputs are set to zero, exploiting the fact that when a single PE is forced to zero, the impact on classification accuracy is negligible [7], [8]. This enables deterministic scheduling of the testing of every PE.

Rather than relying on logic Built-In Self Test (BIST) to perform on-line testing, we propose an approach based on functional test. The inputs of the PE under test are connected to those of its neighbour, as shown in red in Figure 3. After computation of a partial sum, an external comparator checks the result of the PE under test with that of its neighbour. If the outputs don't match, the PE under test is taken offline, meaning its external outputs remain set to zero. Once a PE is tested, the next PE starts the test procedure. This finegrained scheduling minimizes detection time, quickly detects the highly impacting SA1 faults and has no impact on the latency of the calculation.

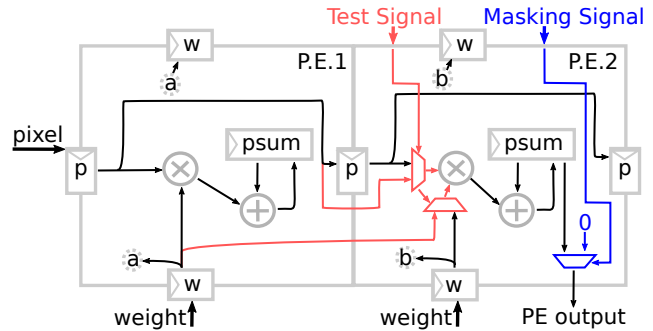

Fig. 3. Schematic of MOZART PE Showing Added Circuitry for Fault Fault Detection (red) and Output Masking (blue)

A transient fault in a PE could result in a mis-match and thus needlessly take the PE off-line. This is prevented by including all PEs in the periodic test procedure, even those that are offline. If an off-line PE shows no further errors after multiple test iterations, it is then taken back on-line, ensuring that transient faults don't result in the loss of PEs.

We have synthesized a 16x16 PE array, with 8-bit integer multipliers, 32 bit adders, and registers for the partial sums. Synthesis results show that the area overhead of the extra test logic is under $8 \%$.

When a PE is found to be faulty, its external output is set to zero, which in DNN applications produces only a minor loss of accuracy.

\section{Output Stationary Architecture for Fault-Tolerant Training}

Dropout is a technique commonly used during training to prevent over-fitting. It consists of setting to zero the value of randomly selected neurons in the last layers of the network. This feature is available in all major DNN frameworks. Some authors [9] have shown that dropout, during training, is effective at improving the fault tolerance of a DNN during inference, however this study was done on an abstract network using a small data-set.

PEs in an accelerator do not map directly to neurons, especially in the case of RS and WS architectures, thus limiting the ability of dropout to mitigate hardware faults. In an OS architecture, the sum computed in an abstract neuron maps to a single PE, however, the PE is re-used to calculate multiple neurons. The OS architecture makes it is possible to exploit dropout to mitigate faults during inference. We applied the dropout process to all the layers in the abstract network.

The combination of detecting and masking faulty PEs to zero with modified dropout during training ensures that, the classification accuracy is minimally impacted in the presence of faults. It is important to note that the proposed dropout technique does not require a priori knowledge of the location of the faults nor does it result in a degradation of accuracy, in the absence of faults.

\section{CASE Study}

To evaluate the MOZART architecture, we have performed a fault-injection study on three different DNNs which have been mapped to multiple systolic architectures. 


\section{A. Selected DNNs}

We have chosen three networks for our experimental study. Two of the networks classify 50,000 images from the ImageNet test data-set into 1000 categories. Top-1 accuracy indicates whether the top ranked category is correct. Top-5 accuracy indicates that the correct category is among the five top-ranked categories, and this is the metric we have used to evaluate the classification accuracy. One modern and compact network, SqueezeNet [10], has been selected as our primary test case, as it is representative of the networks used in embedded applications. Since many, previous fault tolerance studies [7], [9], [11] have used VGG-16 [12] and LeNet-5, we have also included these two networks. VGG-16 is a large network with a huge number of weights, thus it inherently has more redundancy. LeNet-5 is a small network that uses the MNIST data-set for the recognition of hand-written digits and is no longer representative of modern applications. With LeNet-5, Top-1 accuracy is used as there are only ten categories.

These networks are summarized in Table I. An 8-bit integer format was chosen, as this data representation has proven to be more robust than floating point, while maintaining a minimal loss in accuracy [13].

TABLE I

CHARACTERISTICS AND ACCURACY OF SELECTED Networks

\begin{tabular}{|c|c|c|c|c|c|}
\hline Network & $\begin{array}{c}\text { Num. } \\
\text { MACs }\end{array}$ & $\begin{array}{c}\text { Num. } \\
\text { Weights }\end{array}$ & Dataset & $\begin{array}{c}\text { Top-1 } \\
\text { Accuracy }\end{array}$ & $\begin{array}{c}\text { Top-5 } \\
\text { Accuracy }\end{array}$ \\
\hline SqueezeNet & $352 \mathrm{M}$ & $1.2 \mathrm{M}$ & ImageNet & $55 \%$ & $75 \%$ \\
VGG-16 & $15 \mathrm{G}$ & $138 \mathrm{M}$ & ImageNet & $70 \%$ & $91 \%$ \\
LeNet-5 & $341 \mathrm{~K}$ & $60 \mathrm{~K}$ & MNIST & $99 \%$ & - \\
\hline
\end{tabular}

\section{B. Hardware Fault Model}

In this study we focus on computational faults occurring at the PE level. Therefore, we define a fault model that covers stuck-at faults affecting the outputs of the PE, as shown Figure 4. Our results thus hold, regardless of the specific implementation of the PE.

The focus of our study is an improved understanding of the propagation of data-path faults, therefore we have adopted a high-level fault-model. We do not claim that this model reflects all possible logic errors occurring in a PE, however, we assert that it is sufficient to compare the propagation of faults between PEs in the three different systolic architectures.

Previous works have studied faults in the DNN weights and PE register files [11], [14], [15] which can be protected with parity or ECC. Protecting the computational logic in the $\mathrm{PE}$ is more difficult, as it requires fault tolerance strategies that typically have high area costs, timing penalties and are architecture dependent.

For the WS and RS architectures, we only inject faults in the 8 Most Significant Bits (MSbits) of the partial sum, because the 20 Least Significant Bits (LSBits) are inherently discarded in the data-path of this architecture. A fault in the LSBits could carry into the MSBits, however, to be able to fairly compare results, we wanted to have the same number of faulty bits (8),

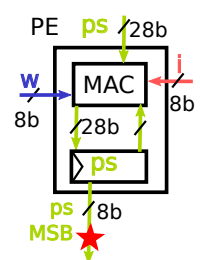

(a) Output Stationary

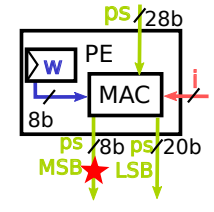

(b) Weight Stationary

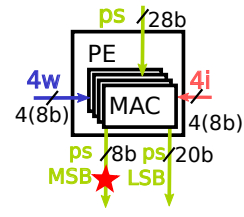

(c) Row Stationary Fig. 4. PE Fault Models in OS, WS and RS Architectures

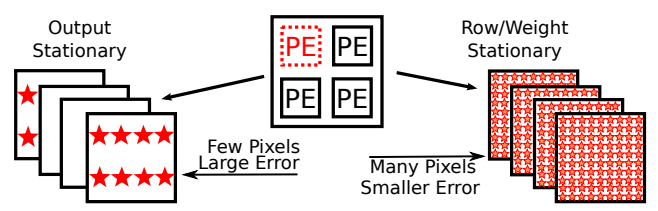

Fig. 5. Propagation of a Fault in a PE in Different Architectures

in all our experiments. Furthermore, as shown later, the LSBits are not very sensitive.

\section{Tool Flow}

We used the N2D2 open-source neural network framework [16] for performing the experiments. It has support for quantized networks and the ability to output a 'C' model. By modifying the ' $\mathrm{C}$ ' model we emulated the effect of faults in the PEs, injecting the types of faults described in Sec III-B.

\section{EXPERIMENTAL RESULTS}

This section presents the experimental results and is organized as follows. Sec. IV-A presents the impact of faults on the three architectures, without mitigation. Sec. IV-B shows the effectiveness of the functional test strategy. Sec. IV-C shows the behaviour of the networks when PEs are masked to zero. Sec. IV-D analyzes the efficiency of the dropout technique. Sec. IV-E demonstrates the scalability of the approach.

\section{A. Impact of Architecture}

Before considering any mitigation techniques, we studied the inherent fault tolerance of the three different architectures. In an OS data-flow, each neuron is mapped to a single PE. However, due to folding, which is required to fit the input map into the PE array, and the fact that the PE array is reused for multiple channels and layers, a given PE is re-used for computing multiple neurons. When there is a fault in a PE, it affects a limited set of specific neurons.

On the other hand, in a WS architecture, each PE contributes to the computation of every neuron for a channel. In this architecture a fault in a PE propagates to all the neurons for an output channel. As only one term in the sum is affected, the numerical impact of the fault is lower.

In a RS architecture, a fault in a PE impacts the result of a 1-D convolution, propagates to multiple output pixels and it is thus similar to a WS architecture. This fault propagation behaviour is illustrated in Figure 5.

We have performed a series of fault injections to study the fault propagation process and assess the fault tolerance of the three architectures. For this analysis, we have limited 


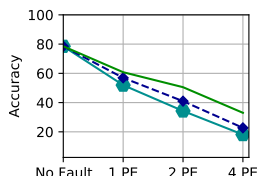

(a) SqueezeNet

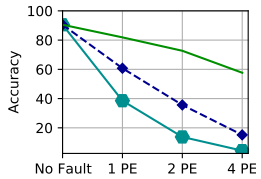

(b) VGG-16

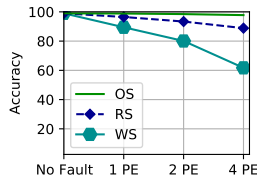

(c) LeNet-5
Fig. 6. Accuracy of Three Systolic Architectures (OS, RS, WS) in the Presence of a Single Faulty Bit

the fault injection scenarios from one to four PEs, as beyond this number, the drop in accuracy is unacceptable. For each data point on the graphs, 1000 randomly selected PEs and bit positions were selected and then 100 randomly selected images were analyzed in order to evaluate the top-5 classification accuracy (top-1 for LeNet-5). The results are summarized in Figure 6 and we see that in all cases, the OS architecture has a higher accuracy. Note, when not stated otherwise, we present results for an array of 256 PEs.

\section{B. Effectiveness of On-Line Test}

A key element of the MOZART approach is on-line testing, which continuously checks the sanity of the PEs, as stuck-at1 (SA1) faults on a single erroneous PE can severely impact the accuracy. The main idea is to sequentially take individual PEs out of the computation and compare the result with their neighbour's. The PE under test is advanced each round of calculation, that is, the computation of the neurons in the array completes and the array is reloaded. For VGG-16, to compute a single image, the array is loaded 55040 times.

To evaluate the detection capability of this technique, we injected SA0, SA1 fault injections on every bit of the outputs of each of the 256 PEs (4096 faults). For each fault, 100 randomly selected images were evaluated to see if the fault could be detected by comparing the partial sum of the PE with that of its neighbour. Table II shows the percentage of all SA1 faults detected after a given number of images.

TABLE II

Percentage of SA1 FAults Detected After N IMAGES

\begin{tabular}{|c|c|c|c|c|}
\hline Percentage of SA1 Faults & \multicolumn{4}{|c|}{ Number of Images } \\
\cline { 2 - 5 } Detected After N Images & 1 & 2 & 4 & 8 \\
\hline SqueezeNet & $100 \%$ & $100 \%$ & $100 \%$ & $100 \%$ \\
VGG-16 & $100 \%$ & $100 \%$ & $100 \%$ & $100 \%$ \\
LeNet-5 & $94 \%$ & $98 \%$ & $99 \%$ & $100 \%$ \\
\hline
\end{tabular}

For the larger networks, a single image was sufficient to detect all the SA1 faults. This was true for any of the 100 random images we used as stimulus for VGG16 and SqueezeNet. For LeNet- 5 with a single image, $94 \%$ of the SA1 faults could be detected, which is already a good level of coverage. In fact, for LeNet-5, more images are required because, with a small network, the number of operations performed per image is is small (see Table I). These experiments show that the lowoverhead approach to on-line test can quickly detect the highly impacting SA1 faults.

Detecting SA0 faults is more difficult because the neuron output values are often zero, especially the MSBits of the integer representation. In Figure 7a, for Squeezenet, we show
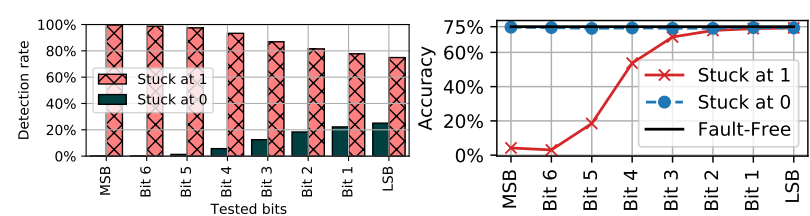

(a) Detection Rate after One Round(b) Accuracy Impact of Single Bit Fault

Fig. 7. Detection Rate and Accuracy with Single Bit SA Faults on Squeezenet

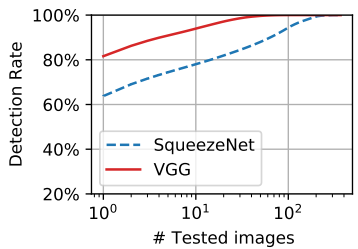

(a) Average

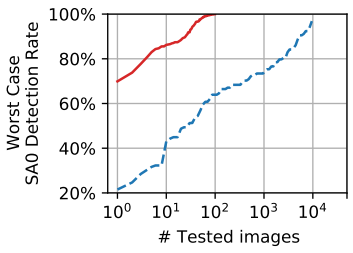

(b) Worst case
Fig. 8. Number of Images Required to Detect Latent SA0 Faults

the fraction of SA faults that were detected after a single round of computation. SA1 faults are easily detected, especially in the MSBits. Conversely, SA0 faults in the MSBits are hard to detect. This is not an important issue, as a single SA0 fault has a negligible impact on classification accuracy, as shown in Figure 7b. These results are consistent with [8], [14].

To understand if SA0 faults can be detected with functional test, we performed two further experiments, focusing on Squeezenet and VGG. First, we considered the 2048 possible SA0 faults, and for each of these faults, we applied 100 randomly selected images. In Figure 8a, we report the percentage of these faults that are detected after a given number of images. We see that after 100 images, all SA0 faults were detected for VGG-16 and 94\% are detected for Squeezenet. This difference can be explained since in VGG-16 a typical PE is tested 215 times for every input image, versus only 42 for Squeezenet. With LeNet-5, as the 16x16 PE array is under-utilized, the SA0 detection is poor and not presented.

To obtain a bound on the worst case number of images required to detect any SA0 faults in Squeezenet, we performed a second experiment. For every possible SA0 fault, 10,000 images were evaluated. We then ranked the images from the one which detected the fewest faults to the one that detected the most. In Figure $8 \mathrm{~b}$ we present the cumulative detection rate, starting on the left with the image with the lowest coverage. Thus, from this graph, we can observe a worst case detection time for SA0 faults. After 10,000 images, we succeed in detecting $97 \%$ of the SA0 faults, which is reasonable, given that these faults are benign.

One might argue that with a more accurate fault model (eg. faults inside the arithmetic logic of the MAC), by relying on the incoming images as stimuli for the on-line testing, there is a risk of latent faults not being detected. If such faults exist, and if the current, real-world stimuli do not activate them, they are not immediately of concern. If the stimulus changes, and the previously latent fault is activated, then the fault is quickly detected by our continuous on-line testing. 


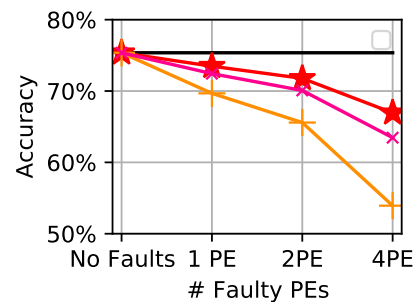

(a) SqueezeNet

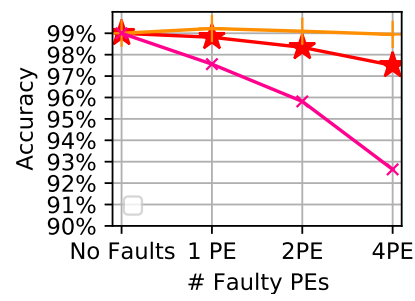

(c) LeNet-5

Fig. 9. Average Accuracy with PEs Masked to Zero

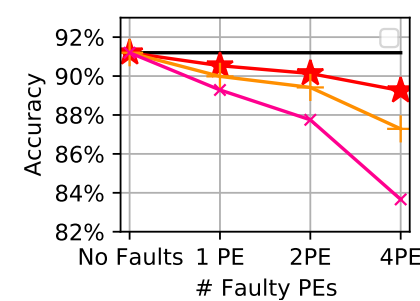

(b) VGG-16

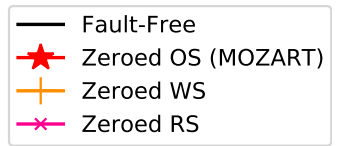

\section{Fault Masking}

In the previous section, we have discussed the procedure for on-line testing. To ensure a fault tolerant design, after detecting a faulty PE, we suppress the potentially serious impact of the fault by forcing the outputs of the faulty PE to zero, removing it from subsequent computations.

1) Average Accuracy: In Figures 9a to $9 \mathrm{c}$ we show the average classification accuracy when the output of one, two or four PEs is masked to zero, for each of the three architectures (OS,WS,RS).

A key point is that for these networks, the output masking technique is most effective for the OS architecture and for Squeezenet and VGG-16, the drop in accuracy when a single PE's output is masked is very small. As more PEs are masked, with the OS architecture, the loss in accuracy is gradual.

LeNet5 has only 10 neurons in the output layer so the impact of faults in this layer has a high probability of corrupting the final result. In this case the OS architecture is more sensitive but the loss in accuracy with 4 masked PEs is only $1.5 \%$.

2) Worst Case Accuracy: Figure 9 only shows the average accuracy. To study the worst-case, we performed a second series of experiments. We generated $\approx 100,000$ groups of 100 randomly selected images and analyzed the accuracy with each architecture. For the unprotected architectures, we injected random faults (1,2 or 4 PEs, with 1 SA faults). For the architectures protected with zero masking, we set to zero 1,2 or 4 PEs. In Table III, we report the minimal observed accuracy across all the groups. First, note that with the unprotected architectures, in the worst case, the accuracy drops to zero for the large networks, even with faults on a single PE. This is a key observation for safety applications.

The second observation is that, with zero masking, the worst case accuracy of the MOZART architecture is significantly better than RS and WS, especially for SqueezeNet. With WS and RS architectures, there exist certain faults that cause a drastic drop in accuracy, unlike the OS architecture which limits the extent of propagation, as illustrated in Figure 5. This is an important take away when designing fault tolerant systolic accelerators.

TABLE III

Worst CASE ACCURACY FOR GROUPS OF 100 IMAGES (HIGHER IS BETTER)

\begin{tabular}{|c|c|c|c|c|c|c|c|c|}
\hline & Network & \multicolumn{4}{|c|}{ Unprotected } & \multicolumn{3}{|c|}{ Zero Masking } \\
\hline $\begin{array}{l}\text { Fault } \\
\text { Type }\end{array}$ & & $\begin{array}{l}\text { Fault } \\
\text { Free }\end{array}$ & OS & WS & $\mathrm{RS}$ & $\begin{array}{c}\text { OS } \\
\text { (Mozart) }\end{array}$ & WS & $\mathrm{RS}$ \\
\hline \multirow{3}{*}{$1 \mathrm{PE}$} & SqueezeNet & 64 & 0 & 0 & 0 & 58 & 2 & 52 \\
\hline & VGG & 82 & 0 & 0 & 0 & 82 & 82 & 79 \\
\hline & Lenet & 95 & 5 & 5 & 4 & 95 & 96 & 77 \\
\hline \multirow{3}{*}{2 PEs } & SqueezeNet & 64 & 0 & 0 & 0 & 53 & 1 & 45 \\
\hline & VGG & 82 & 0 & 0 & 0 & 80 & 77 & 74 \\
\hline & Lenet & 95 & 2 & 3 & 2 & 92 & 95 & 72 \\
\hline \multirow{3}{*}{4 PEs } & SqueezeNet & 64 & 0 & 0 & 0 & 53 & 0 & 16 \\
\hline & VGG & 82 & 0 & 0 & 0 & 79 & 73 & 63 \\
\hline & Lenet & 95 & 4 & 2 & 2 & 90 & 95 & 58 \\
\hline
\end{tabular}

\section{Dropout During Training}

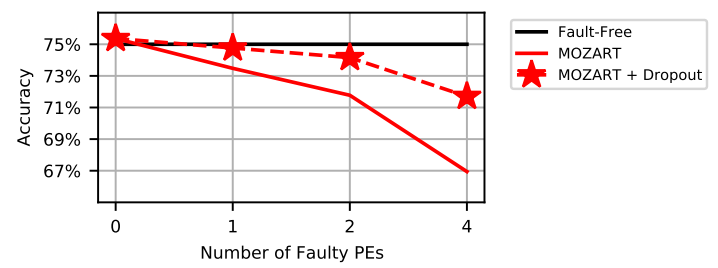

Fig. 10. Squeezenet Accuracy with Faulty PEs when Trained with Dropout

Dropout during training can be used to improve the robustness during inference. We trained Squeezenet with 5\% dropout on all layers, whereas typically dropout is only applied to the last layers. The training time increased $(\approx 3 x)$, but the resulting configuration provided additional fault tolerance, as shown in Figure 10. Using the newly trained network and a MOZART architecture, we injected single bit faults in a varying number of PEs. With faults in 4 PEs, due to the new training, the accuracy increased from $67 \%$ to $72 \%$. This modified training was performed once, with no knowledge of the faults. This is different from [7], where the re-training was done based on knowing the position of the faults.

\section{E. Scalability}

Up to now, data has been presented for a $256 \mathrm{PE}(16 \mathrm{x} 16)$ array. We tested the MOZART architecture for different PE array sizes and the results are shown in Figure 11. These results are for Squeezenet with a single SA fault on a single PE. As expected, when the array size increases, the impact of a single faulty PE is reduced. In all cases, MOZART provides a significant improvement.

\section{RELATED WORK}

Historically, the fault tolerance of systolic arrays has been studied with the objective that the final computed result be identical in the presence of a fault [17]. For DNNs, this constraint can be relaxed, since classification accuracy is never perfect and the requirement is only that it remain acceptable.

Until recently, most studies of DNN fault tolerance worked with an abstract model of the network, independent of the 


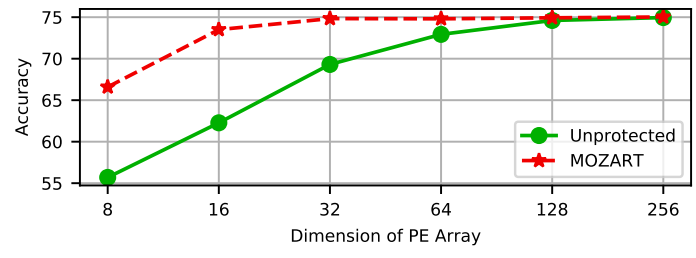

Fig. 11. Squeezenet Accuracy for Varying Array Sizes (1 SA fault on $1 \mathrm{PE}$ )

hardware. In [11], the authors perform a sensitivity study of three networks applying bit flips in the weights. In the Ares framework [14], faults are injected in the weights and activations of the abstract model. In [13], the authors study GPU-based DNNs and conclude that small, integer numeric formats result in increased robustness compared to floating point. An in-depth study of fault injections on the RTL model of an accelerator mapped to a FPGA is presented in [18]. It is shown that SA0 faults have minimal impact on accuracy but their study is limited to a small data-set.

In [19], the authors propose an opportunistic approach for on-line testing of DNN accelerators, exploiting the fact that, due to folding, some PEs are temporarily idle. Since not all PEs are tested, they can can not guarantee coverage, which is a requirement for safety standards.

In [8], the authors propose a fault tolerant DNN accelerator using Razor techniques to detect timing faults. The main focus is low voltage operation, not safety applications.

The work of Zhang [7] is the closest to that presented in this paper. They propose fault mitigation for a WS accelerator, using a multiplexer to mask the output of the erroneous multiplier of a PE. They also propose Fault-Aware Pruning(FAP). Their results are interesting, but have certain limitations. First, they have chosen a WS Architecture, which does not limit fault propagation. Also, the FAP techniques requires knowledge of the hardware faults prior to training. Finally, they propose no test technique to detect faults and only study LeNet-5, which is not representative of modern DNNs.

\section{CONCLUSiOnS}

Autonomous systems that rely on DNNs must meet safety requirements. To address this need, we have presented the MOZART approach for a fault tolerant DNN accelerator. We analyzed the robustness of the three most common systolic architectures used in DNN accelerators (OS,WS and RS), a study which has not been performed previously and show that the OS architecture inherently limits the propagation of faults.

The second aspect of MOZART, is an on-line fault detection scheme, based on temporarily taking PEs out of service to compare their results with their neighbours. Using this technique, within the time required to process a single image, all the SA1 faults can be detected for the two large networks. Fast fault detection is a requirement for safety and we achieve this with low hardware overhead.

Third, we have shown that connecting the output of a PE to zero to mask known faults is particularly effective with the OS architecture. We not only analyzed the average classification accuracy for a large number of faults, as is common in other studies, we also measured a worst case accuracy when groups of images are analyzed. A key take away, is that there exist pathological faults which can cause the worst case accuracy to drop close to zero. This is an important message, and future studies of fault tolerance in DNNs should consider this metric.

Other authors have shown that DNNs can be trained to perform well when a set of known faults are present but this is of little practical value, as it would require massive compute resources to perform a customized training for each device. We have shown that dropout applied to all layers during training, increases fault tolerance, regardless of which PE is faulty.

A full safety analysis of an integrated circuit is beyond the scope of a scientific paper. It would require considering technology specific faults and analyzing the full design including control logic. Nonetheless, the concepts we presented constitute innovative safety mechanisms, applicable to the architecture and design of the data-path of systolic DNN accelerators.

\section{REFERENCES}

[1] A. Reuther et al., "Survey and Benchmarking of Machine Learning Accelerators." IEEE, Sep. 2019, pp. 1-9.

[2] V. Sze et al., "Efficient Processing of Deep Neural Networks: A Tutorial and Survey," Proceedings of the IEEE, vol. 105, no. 12, Dec. 2017.

[3] G. Li et al., "Understanding error propagation in deep learning neural network (DNN) accelerators and applications." ACM Press, 2017.

[4] S. Park et al., "4.6 A1.93tops/W scalable deep learning/inference processor with tetra-parallel MIMD architecture for big-data applications." IEEE, Feb. 2015, pp. 1-3.

[5] Z. Du et al., "ShiDianNao: shifting vision processing closer to the sensor." ACM Press, 2015, pp. 92-104.

[6] Y.-H. Chen et al., "Eyeriss: A Spatial Architecture for Energy-Efficient Dataflow for Convolutional Neural Networks." IEEE, Jun. 2016, pp. 367-379.

[7] J. J. Zhang et al., "Fault-Tolerant Systolic Array Based Accelerators for Deep Neural Network Execution," IEEE Design \& Test, vol. 36, no. 5, pp. 44-53, Oct. 2019.

[8] B. Reagen et al., "Minerva: Enabling Low-Power, Highly-Accurate Deep Neural Network Accelerators." IEEE, Jun. 2016, pp. 267-278.

[9] R. A. Solovyev et al., "Study of Fault Tolerance Methods for Hardware Implementations of Convolutional Neural Networks," Optical Memory and Neural Networks, vol. 28, no. 2, pp. 82-88, Apr. 2019.

[10] F. Iandola and K. Keutzer, "Keynote: small neural nets are beautiful: enabling embedded systems with small deep-neural- network architectures," in 2017 International Conference on Hardware/Software Codesign and System Synthesis (CODES+ISSS), Oct 2017, pp. 1-10.

[11] Arechiga and Michaels, The Robustness of Modern Deep Learning Architectures against Single Event Upset Errors, 2019, 67.

[12] K. Simonyan and A. Zisserman, "Very deep convolutional networks for large-scale image recognition," in 3rd International Conference on Learning Representations, ICLR 2015, 2015.

[13] F. F. dos Santos et al., "Impact of Reduced Precision in the Reliability of Deep Neural Networks for Object Detection." IEEE, May 2019.

[14] B. Reagen et al., "Ares: A framework for quantifying the resilience of deep neural networks." IEEE, Jun. 2018, pp. 1-6.

[15] S. Kim et al., "Matic: Learning around errors for efficient low-voltage neural network accelerators," in 2018 Design, Automation Test in Europe Conference Exhibition (DATE), 2018, pp. 1-6.

[16] O. Bichler, "N2d2," https://github.com/CEA-LIST/N2D2.

[17] J. H. Kim and S. M. Reddy, "On the design of fault-tolerant twodimensional systolic arrays for yield enhancement," IEEE Transactions on Computers, vol. 38, no. 4, pp. 515-525, 1989.

[18] B. Salami et al., "On the resilience of RTL NN accelerators: Fault characterization and mitigation," in IEEE SBAC-PAD.

[19] W. Li et al., "Soft Error Mitigation for Deep Convolution Neural Network on FPGA Accelerators." IEEE, Aug. 2020, pp. 1-5. 